\title{
La educación en la sociedad multicultural
}

\author{
M ariano Sánchez ${ }^{1}$ \\ M aría Jesús 0 rdóñez ${ }^{2}$ \\ 1. Universidad de Granada. D epartamento de Sociología. \\ Rector López Argüeta, s/n. 18071 G ranada. Sapin \\ 2. Universidad de California
}

\section{Resumen}

El aprender, en cuanto a experiencia condicionada por la acción recíproca entre la persona y el mundo, puede ser considerado desde un punto de vista biológico y desde un punto de vista sociocultural. Las ideas del mundo se desarrollan en distintas condiciones, el clima, las razas, las naciones determinadas por la historia y la formación de los Estados, las delimitaciones, condicionadas según las épocas, se enlazan con las condiciones especiales que influyen en el origen de la multiplicidad de las visiones del mundo. La diferencias entre ser, poder y fin del hombre hace necesaria la educación: una acción conjunta de los individuos con el objeto de ayudar a los recién nacidos, a los niños y niñas y adolescentes a aprender la «vida humana».

La educación y su investigación, así como la reflexión crítica sobre la misma, adquieren hoy día mayor importancia, ya que la forma de vida del individuo, así como las formas de convivencia social, dependen cada vez más de soluciones a conflictos de creencias y de cultura que se deben plantear en la educación.

El campo propio de la educación sería el de la libertad, como condición imprescindible para un libre desenvolvimiento de la personalidad. La educación de personas libres, en cuanto no puede tener otro fin que el desenvolvimiento libre de la personalidad, rechaza toda coacción. Tendríamos así un modelo de educación en el que el ideal que la define se concreta en el desarrollo de personas libres, pues la característica de la educación en la modernidad consiste en suprimir la coacción.

El currículum multicultural exige un marco democrático de decisiones sobre los contenidos de la enseñanza para representar los intereses de todos. Se trata de un desafío: lograr que la diversidad cultural entre personas y colectivos, etc. deje de ser motivo de marginación para ser factor de enriquecimiento humano.

Palabras clave: socialización, instrucción, multiculturalidad, sociología, educación.

Abstract. The education in the multicultural society

It is possible to consider learning with respect to a process conditioned by a reciprocal action between a person and the world. I deas of the world are developing under different conditions, climates, races, nations determined by history and formation of countries, delimitation's determined by time periods connected with special conditions of influence whose origin is the diversity of views of the world. Education is necessary to differentiate between to be, to be able to and purpose of man: an ensemble action of people with the objective 
to help new borns, children and adolescents learn «human life». Education and its investigation following in the same line as critical thought, are of greatest importance today because of real people, manners of socializing depend more and more on solutions to cultural conflicts that must be posed to education. The field of education could be that of freedom as an essential condition for the free development of personality. Education of free people, which can not have other aims than the free development of people, lies in the elimination of all coercion. The result would b an education «model» whose ideals are defined by the development of free people, since the characteristic of education in modern times is based on eliminating coercion.

The multicultural curriculum needs a democratic program to make the best decisions about the academic contents to represent everyone's interests. This the a challenge: Suceed in making the cultural diversity between people and groups, etc. not a cause of exclusion but a factor of human enrichment.

Key words: socialization, instruction, multiculturality, sociology, education.

\section{Sumario}

N uevo planteamiento de la cultura escolar
La relación entre la naturaleza y la cultura Bibliografía

La educación es un aprendizaje social de mayor complejidad que la socialización y la instrucción que implica características de ambas; sin embargo, el concepto más amplio es el de socialización porque las prácticas educativas suponen un tipo particular de socialización (Lerena, 1988: 111). Para el individuo, la socialización supone aprendizaje, «interiorización de» y cierta «adaptación a» su entorno sociocultural; en cambio, para el grupo, este mismo proceso se interpreta mejor como una forma de control por la producción social de la identidad de sus miembros mediante la transmisión, de manera inconsciente y no formalizada, de los conocimientos, valores, hábitos y actitudes que constituyen la cultura básica de una sociedad. «EI orden social existente se mantiene en gran parte con la interiorización por el individuo de las normas sociales, mucho más que por la intervención de mecanismos represivos o la coacción. Con la socialización, [... ] el individuo se conforma a expectativas sociales sin tener una percepción consciente, a menudo, de estar sometido a normas socia les» (I glesias, 1988: 169). La instrucción, en cambio, es un proceso formalizado que transmite los conocimientos generales (leer, escribir, hablar y nociones básicas de las ciencias). La generalización de la instrucción es uno de los índices que distingue a la sociedad moderna de la tradicional, pues, el tanto por ciento de la población que ha recibido instrucción es un indicador importante del grado de modernización alcanzado.

La educación se produce de manera informal pero consciente en la familia y en la sociedad y de manera formal en las instituciones de enseñanza. ¿C ómo se diferencia de la socialización y la instrucción? Su finalidad es la realización de un tipo ideal de individuo, en ese sentido se ha dicho que «la educación no 
educa, hace algo mucho más esencial. No la educación, sino, sociológicamente hablando, la práctica educativa [... ] contribuye a crear particulares tipos humanos, individualidades, conciencias» (Lerena, 1988: 106). Porque la educación implica la referencia a unos valores, creencias, presupone una antropología, una cosmovisión que definen una determinada cultura. La diferencia entre ser, poder y fin del individuo hace necesaria la educación con el objeto de ayudar a los recién nacidos, a los niños y niñas y adolescentes a aprender la «vida humana».

En la pedagogía o teoría educativa se distinguen tres generaciones representadas por diversos autores, en la primera generación: N ohl, Spranger; en la segunda generación: Flitner, G eissler, Blochmann. Con los siguientes antecedentes: 1의 «filosofía de la vida» de D ilthey o teoría de las ciencias del espíritu; 2o) tradición neoeuropea del pensamiento pedagógico: Rousseau, Pestalozzi, H erbart, Fröebel; en la tercera generación: pedagogía científica o ciencia de la educación crítico constructiva. Según D ilthey: «La educación es la actividad planeada por la cual los profesores forman la vida anímica de los seres en desarrollo». Para Flitner: «La educación es el proceso de crecimiento y maduración de los jóvenes dentro de unas etapas en las cuales los adultos protegen y fomentan el mencionado proceso». Para Fröebel la educación consiste en: «Suscitar las energías del hombre como ser progresivamente consciente, pensante e inteligente, ayudarle a manifestar con toda pureza y perfección, con espontaneidad y conciencia, su ley interior, lo divino que hay en él; en esto consiste la educación del hombre». Según Pestal ozzi: «La educación es el desarrollo natural, progresivo y sistemático de todas las facultades».

En la sociedad contemporánea el paradigma educativo tiene como uno de sus fundamentos la diversidad cultural. La educación debe transmitir los valores que implica la convivencia plural: libertad, tolerancia, respeto y favorecer el diálogo y el acuerdo, característicos de la pedagogía crítica. La reflexión crítica sobre la educación es importante, ya que la forma de vida del individuo, así como las formas de convivencia social, dependen cada vez más de soluciones a conflictos que plantea la diversidad cultural (Speck, 1981: 595). La educación, como medio fundamental de socialización, debe investigar la realidad multicultural en las sociedades democráticas.

\section{N uevo planteamiento de la cultura escolar}

Pedagogía es la ciencia social que estudia la conexión y las características de los conceptos de socialización, instrucción y educación. ¿Es la coacción un rasgo común del aprendizaje en el contexto social y escolar? (Sotelo, 1995: 37). La coacción parece un componente en la socialización, ya que el grupo presiona para que se sigan sus normas y de ello se derive la integración al asimilarlas sin cuestionarlas. Sin embargo, deberíamos preguntarnos ¿qué espacio quedaría entonces para el cambio, para la innovación? La tradición educativa demuestra que los elementos coactivos están presentes en la educación; las prácticas educativas son «relaciones de imposición y dominación» (Lerena, 
1988: 119). Pero es posible hablar de libertad en la práctica educativa si defendemos la posibilidad de resistencia individual ante la coacción, la imposición, el poder y adoptamos una visión distinta. El fundamento de la educación podría ser la libertad necesaria para la real ización personal. En la modernidad, el ideal de educación se concreta en el desarrollo libre de personas libres; el pluralismo de las concepciones del mundo y de ideales en la sociedad contemporánea obliga a un nuevo planteamiento de la cultura escolar, no es posible un concepto de educación universal mente aceptado.

Las ideas del mundo se desarrollan en distintas condiciones, el clima, las razas, las naciones determinadas por la historia y la formación de los Estados, las delimitaciones condicionadas según las épocas se enlazan con las condiciones especiales que influyen en el origen de la multiplicidad de las visiones del mundo (Dilthey, 1988: 47). La propuesta de una educación multicultural no es nueva, en realidad, los intercambios y el influjo de unos países sobre otros ha sido una constante de la historia europea; todos los países europeos tienen establecidas políticas concretas para la integración cultural de los inmigrantes y para respetar su identidad cultural, así como para luchar contra el racismo. En la recomendación núm. 14 del Libro verde sobre la dimensión europea de la educación, presentado en 1993 por la Comisión de la Comunidad Europea, se destaca como «la atención que debe prestarse al respeto a las identidades y de las diferencias culturales y étnicas, y la importancia de luchar contra toda forma de chauvinismo y de xenofobia, son componentes esenciales de la acción educativa». Subrayando este significado, en la propuesta 29 se especifica: «La introducción de dicha dimensión requiere que los profesores: Aprendan a compartir y a hacer compartir la riqueza de las culturas europeas. Fomenten una identidad europea paralela a las identidades nacionales y regionales». Y la recomendación 15 precisa que «los sistemas educativos no deben limitarse únicamente a transmitir conocimientos, sino que deben formar también a los jóvenes en el espíritu de la democracia, de la lucha contra la desigualdad, de la tole rancia y del respeto a la diversidad». En el art. 126 del Tratado de la U nión Europea se considera importante iniciar una reflexión previa sobre la finalidad y los medios que permitan a la Comunidad «contribuir al desarrollo de una educación de calidad fomentando la cooperación entre los Estados miembros y, si fuera necesario, apoyando y completando la acción de éstos en el pleno respeto de sus responsabilidades en cuanto a los contenidos de la enseñanza y a la organización del sistema educativo, así como de su diversidad cultural y lingüística».

El currículum multicultural «exige un marco democrático de decisiones sobre los contenidos de la enseñanza en el que los intereses de todos queden representados» (Gimeno, 1991). ¿Acaso un «marco democrático» garantiza la representación de todos los intereses? No olvidemos que cualquier actor social, más aún si tiene poder, puede desplegar estrategias de cultivo del pluralismo con la pretensión última de continuar controlando una realidad. M ientras los sistemas - los «marcos» - democráticos crecen, también lo hace la práctica de la intolerancia; en opinión de Pastor (1994: 125) «es uno de los puntos más 
débiles en todas las democracias formales del mundo occidental: la práctica o el respeto factual del pluriculturalismo. Una verdadera profundización democrática debería asumir la realidad social pluricultural, afirmando positivamente el valor de todos los miembros de la sociedad». La creciente complejidad de la sociedad moderna, la inclusión de grupos étnicos minoritarios y las posibles actitudes racistas en la población explican el interés por el tema en la educación. Corresponde a la escuela, y más exactamentea la enseñanza de las ciencias humanas, dominar nuestro primer impulso propenso a la «negación del otro» (Finkielkraut, 1987: 100); pero aunqueel relativismo plantea la tolerancia entre culturas autónomas, se trata de una visión estática de la cultura que no permite una verdadera aceptación de la diversidad. A partir de una concepción diná mica de la variedad cultural podríamos desechar el concepto asimilacionista que implica proponer como modelo válido el de un sector social de la sociedad mayor y también liberar de la necesidad de considerar a las culturas de origen de las minorías como estáticas y reconocer su posibilidad de cambio; el desafío consiste en ver la diferencia cultural no como un obstáculo sino como un factor positivo, porque como afirma M orin (1988: 108), «o que importa en la vida y el devenir de la cultura europea es el encuentro fecundador de las diversidades, los antagonismos, las concurrencias, las complementariedades, es decir, su dialógica». En ese sentido escribió Lerena (1989: 337): «Las culturas de los grupos dominados no están en desventaja con relación a la cultura dominante, no son inferiores, no representan un cúmulo de faltas, sino que representan un universo alternativo». La tolerancia del otro, la «pedagogía de la relatividad» sobre la que escribe Finkielkraut, tiene algunos inconvenientes. El primero, caer en la peor interpretación del ideal igual itarista y el segundo el riesgo de la fragmentación, es decir, con palabras del autor «negar a los hombres de épocas diversas o de civilizaciones alejadas la posibilidad de comunicarse acerca de significados pensables y valores que rebasen el perímetro de donde han salido» (1987: 105). Para superar los problemas del relativismo, Julio Carabaña (1993a: 69) propone la recuperación de la primacía del individuo frente al grupo porque «los principios del individualismo universalista son desde hace un tiempo el principio fundamental de nuestras democracias». Con las siguientes palabras defiende Bernhardt (1994: 16) el concepto más individualista de cultura: «Si mañana queremos convivir y entendernos, tenemos que comprender hoy que la cultura es un bien individual que todos poseemos, que nace y crece en nosotros, [... ] de manera que yo - como individuo - pueda crear por mi propio esfuerzo y el ejercicio de mi libertad una cultura nueva dentro de mí que se prolonga en la unicidad cultural de mi vecino». Para H ell (1994: 126) la idea de cultura implica «la supremacía del hombre sobre el ciudadano».

Palabra clave en el vocabulario de la educación, el término cultura integra en su significado una faceta individual y colectiva, normativa y descriptiva, un carácter universalista y diferencialista; en el significado tradicional, individual y normativo, la cultura se presenta como conjunto de conocimientos y competencias cognitivas generales, considerada como capacidad de evaluación inteligente y de juicio personal en materia intelectual y artística, con un significado 
«promocional», según la expresión de Camilleri (1985). Por otra parte, el significado descriptivo y objetivo desarrollado por las ciencias sociales contemporáneas: la cultura como conjunto de características del modo de vida de una sociedad, de una comunidad o de un grupo que incluye los aspectos considerados más cotidianos; por el hecho de que no todos los componentes de la cultura en el sentido sociológico son de igual valor y utilidad, es por lo que el significado descriptivo de la sociología se cuestiona en la enseñanza y el significado individual y «perfectivo» es parcial. ¿Q ué significa pues la palabra cultura cuando se habla de la función de transmisión cultural de la educación? D e todas las cuestiones planteadas sobre los problemas de la educación, las que tratan de la función de transmisión cultural en las instituciones de enseñanza son de las más importantes; se ocupan del contenido de la práctica en la enseñanza, su interés, su utilidad, cuestionan a los docentes sobre su valor educativo o cultural. El desarrollo de este tipo de cuestiones constituye un factor esencial para definir lo que se ha llamado a partir de la década de los sesenta «crisis de la educación» (Forquin, 1989). H oy el conjunto de los saberes que los griegos llamaban «enkukli os paideia» se ha desbordado, la función de transmisión cultural en la enseñanza es cada vez más difícil de identificar y realizar, por tanto, es necesario pensar sobre el contenido de la cultura en los diferentes tipos de opciones educativas; la cuestión de las implicaciones culturales en la enseñanza es esencial. La transmisión cultural en la enseñanza supone una selección y una producción de contenidos de cultura para educar a las nuevas generaciones. $\mathrm{H}$ ay que reconocer que la cultura no es unitaria e inmutable, sino al con7trario, según la historia y la geografía, varía de una sociedad a otra y de un grupo a otro en la misma sociedad, es decir, la educación no transmite nunca la cultura considerada como un patrimonio simbólico unitario y coherente.

En el pensamiento anglosajón contemporáneo, se subraya el pluralismo de las culturas y desde otra posición se señala la existencia de convergencias, de similitudes más allá de las fronteras étnicas, lingüísticas o ideológicas; estas ideas son importantes en el debate sobre las funciones y las implicaciones culturales de los conocimientos, los significados y los val ores que constituyen el contenido del currículum. En los años sesenta, Raymond W illians, G eoffrey Bantock y Paul H irs (Forquin, 1989) abordan la cuestión de los fundamentos y los valores culturales de la educación a partir de tres postulados radical mente diferentes. Los dos primeros tienen en común preguntarse sobre la calidad de la cultura y sobre los fundamentos y las implicaciones culturales de los mensajes escolares. Para W illiams la perspectiva de una transformación socialista de la sociedad supone el acceso a todos de los elementos fundamentales de la cultura humana mediante el currículum de estudios generales iguales para todos; Bantock desarrolló una concepción pesimista de la generalización del alfabetismo que preconiza un dualismo escolar radical, reservando la cultura superior a una élite. La perspectiva de Paul $\mathrm{H}$ irst fue diferente por las aportaciones de la filosofía analítica y el carácter intemporal de su idea del currículum «liberal» centrado en la condición racionalista del acceso de todos a los dife rentes tipos de pensamiento conceptual. A partir de estas ideas sobre el relati- 
vismo, se plantea cuáles son las aportaciones del multiculturalismo en la teoría de la educación.

El concepto de educación va unido al concepto de valor, «la educación multicultural debe concebirse y practicarse en su interconexión con la "educación para el pluralismo"» (M itter, 1992: 33), la educación multicultural debe responder a la exigencia de elaborar programas pedagógicos que permitan superar, 0 atenuar, el conflicto en torno a los valores de las personas y los grupos interesados. Como señala el filósofo Richard Peters, educar a alguien, no es que él/ella aprenda no importa qué y tampoco es formación en el sentido técnico de training. Educar a alguien es iniciarlo en una cierta categoría de actividades que no tienen un valor instrumental, que no son medios para llegar a otra cosa, como el éxito social, sino un valor intrínseco, un valor que se vincula al hecho mismo de las prácticas (como por ejemplo en el caso del arte) o que trata de favorecer el desarrollo de capacidades y de actitudes que son consideradas como deseables por sí mismas. Es un hecho que los estudiantes de diferentes medios sociales vienen a la escuela con ciertas características culturales que influyen directamente en la forma en que realizan la escolarización; hay que destacar que el factor de desigualdad escolar es una aportación de la sociología que ninguna teoría, filosofía o política de la educación puede ignorar.

Consideramos la cultura un sistema de actitudes, valores, creencias y conocimientos ampliamente compartidos en el seno de una sociedad transmitidos de generación en generación (I nglehart, 1991: 5), la cultura se aprende aunque puede variar de una sociedad a otra, y lo que para nosotros es más importante, puede transmitirse. «El sistema de enseñanza constituye un medio para reproducir la particular cultura de un grupo concreto y relativamente autónomo, depositario y representante de esa cultura» (Lerena, 1988: 126). A la vista de cambios importantes en las condiciones sociales, pueden ser transformadas incluso partes centrales de una cultura, pero es mucho más probable que esto tenga lugar por medio del reemplazo intergeneracional de la población que por medio de la educación de adultos ya socializados. Las diferencias en el aprendizaje cultural también juegan un papel esencial a la hora de determinar lo que la gente piensa y hace. ¿Existen diferencias interculturales estables en las orientaciones básicas de los diferentes pueblos? Para Inglehart (1991) existen diferencias interculturales sustanciales y perdurables en lo que respecta a ciertas actitudes y hábitos básicos de las poblaciones en sociedades determinadas (gitanos, etc.), pero si bien estas diferencias son relativamente esta bles, no son inmutables; se pueden conocer sus causas específicas, entre ellas el desarrollo económico a largo plazo parece ser especialmente importante. Aunque la economía y la cultura política sean distintos grupos de variables, están muy relacionadas. Los cambios económicos influyen en el cambio cultural, pero no son el único factor implicado; los modelos culturales pueden persistir mucho tiempo después que los factores de origen dejan de ser operativos. Algunas variables culturales no sólo se relacionan con el desarrollo económico, sino que parecen tener un papel básico en el establecimiento de la democracia moderna; por ejemplo, la sociedad moderna sólo puede 
funcionar si la gente piensa que los extranjeros no son enemigos porque las empresas y las burocracias a gran escala que hacen posible las modernas organizaciones políticas y económicas dependen de modelos de interacción predecibles y fiables entre personas que son totalmente extraños. Pero, si bien es importante, la confianza interpersonal es evidente que no es suficiente para apoyar una democracia de masas estable.

\section{La relación entre la naturaleza y la cultura}

La enseñanza, en el sentido de aprendizaje sociocultural, se basa en la capacidad de la persona para aprender aquellos aspectos de la cultura necesarios para su formación como individuo y para el desarrollo social. El aprendizaje puede ser considerado desde un punto de vista biológico y desde un punto de vista sociocultural (Correll, 1980: 11). Sobre la hominización, en palabras de M orin y Piatelli (Apostel, 1983: 192), la distribución de los rasgos genéticos en el seno de las poblaciones humanas, la relación entre organismo y medio, las relaciones entre desarrollo cerebral y desarrollo cognoscitivo han revelado la estrecha interdependencia entre estructuras biológicas y comportamiento, descubriendo así que los lazos biosociales, ya observables en las especies infrahumanas, se imbrican unos con otros y se complican en el hombre para convertirse en un lazo biocultural. «La cultura expresa funciones esenciales de la vida, individual o colectiva; no se sobreañade a lo biológico; lo incluye en su dominio» (H ell, 1994: 125). No sólo la filogénesis (crecimiento del cerebro en volumen y en número de circunvoluciones) es inseparable del desarrollo de la cultura (lenguaje, técnica, riqueza de las interacciones entre los individuos), sino que también la ontogénesis de cada individuo se inscribe necesariamente en un «medio» cultural: los rasgos típicamente humanos (posición y andadura erectas, lenguaje, socialización) sólo pueden manifestarse gracias a la interacción de los adultos entre sí, a los cuidados y educación dados al recién nacido por la comunidad y el contexto cultural. El término cultura comprende «la totalidad de las relaciones de la persona con la naturaleza, con la sociedad, consigo mismos como individuos y con la esfera religiosa o metafísica» (M itter, 1992: 31). U na relación en espiral se establece entre natural eza y cultura y determina desde el principio los rasgos esenciales de la evolución humana. Como explica Julio C arabaña (1993b), aunque el hombre, como el resto de los animales, es natural mente social, también es naturalmente cultural - capaz mediante el lenguaje ${ }^{1}$ de crear, acumular y transmitir conocimiento- , aquí se encuentra la característica esencial de la sociedad humana. Aunque la unidad de la especie es fundamental, su diversidad se manifiesta en los individuos, las razas, las etnias, las culturas, las sociedades; la extrema diversidad genética se comprueba de modo particular en los caracteres psicoafectivos de las personas; la internacionalización tecnológica viene a subrayar la identidad de la especie y se afirma que la Tierra misma se ha convertido en una espiral biocultural

1. La música, el arte, etc. 
(spaceship earth). Actualmente hay que hablar de la defensa de las diversidades etnoculturales, mediante el reconocimiento ideológico, político y científico del carácter indisociable de la unidad y de la diversidad de la especie humana. Se trata de «construir la unidad por la diversidad». Porque la etimología de universo, lejos de lo singular, lo que destaca es que lo múltiple, lo plural, se vuelve, se «vierte hacia» la unidad, que adquiere más valor en la diversidad.

L as constituciones y declaraciones de las principales leyes de educación de distintos países ofrecen una experiencia de relativismo cultural. Las finalidades de la educación suelen ser muy semejantes. Sólo la aparición de determinadas palabras en el texto (como socialista, budista, o islámico) señala un determinado sistema de valores; otros términos, como desarrollo o multicultural, se refieren a las estructuras económicas y sociales. Las declaraciones de las finalidades de la educación se convierten en formas operativas concretas mediante las políticas que se aplican en los sistemas de educación. «Las finalidades, metas, objetivos y políticas de la educación son el resultado de la acción de fuerzas sociales, económicas e históricas y expresan las aspiraciones del porvenir» (OIE, 1981: 25). El proceso de educación y socialización, en sí mismo, no exige la existencia de una institución específica, de hecho en las sociedades primitivas los dos procesos coinciden. Con el fin de ofrecer a los jóvenes el conocimiento necesario para determinadas funciones y hacerles aprender Ios ideales y los valores de la sociedad surge la institución de enseñanza. El objeto de la sociología de la educación se ha visto como el análisis de la contribución del sistema educativo a la transmisión de la cultura. Parsons (1974) subraya la continuidad entre la escuela y la familia, «la escuela sirve para hacer interiorizar a los estudiantes esfuerzo y capacidad para el éxito en sus futuros roles adultos, y funciona para situar estos recursos humanos en el interior de la estructura de roles de la sociedad adulta». La educación básica se aprende sobre todo en la familia, la escuela puede incrementar o atenuar las influencias y cuando las influencias son semejantes se produce un efecto acumulativo. La estructura interna del sistema de enseñanza puede cambiar la calidad de la educación; si hay una gran coherencia entre los profesores y/o las profesoras, los programas, los libros de texto, también la influencia será mayor. Esto explica el interés de los diversos grupos de poder en garantizar la conservación de los modelos culturales, la consecución de los fines, la adaptación a las condiciones ambientales, la integración social mediante el sistema educativo.

Por último, recordar que el sistema de enseñanza liberal aparece configurado en la Constitución francesa de 1793, en la cual se reconoce, por primera vez, el derecho universal a la educación y el deber del Estado de ponerla al alcance de todos los ciudadanos; en el decreto de 15 de septiembre del mismo año se concibe el sistema escolar como instrumento de formación de la fuerza de trabajo, y como mecanismo reproductor, en el plano ideológico, de los distintos grupos o clases. A finales del siglo xx diferentes grupos sociales se encuentran excluidos de la cultura mayoritaria y el derecho a la educación favorece la jerarquía de las identidades culturales, contraria «a la absoluta dispersión» (Foucault, 1995) de los sistemas de pensamiento y de las prácticas sociales. 


\section{Bibliografía}

Apostel, L. y otros (1983). La interdisciplinaridad y ciencias humanas. Barcelona: Unesco. Tecnos.

BERN H ARDT, R. (1994). «¿Es posible una cultura europea?» (inédito).

CAMILLERI, C. (1985). Antropología cultural y educación. París: O IE. U nesco.

CARABAÑ A, J. (1993a). «A favor del individualismo y contra las ideologías multiculturales». Revista deEducación, 302. M adrid: M EC.

- (1993b). «D e la conveniencia de no confundir sociedad y cultura». En Problemas de teoría social contemporánea. LAM O DE ESPIN OSA, E.; Rod RíGUEZ IBÁÑNEZ, J.E. $M$ adrid: Centro de Investigaciones Sociológicas.

CORRELL,W. (1980). El aprender. Barcelona: H erder.

DILTHEY, W. (1988). Teorías de las concepciones del mundo. M adrid: Alianza.

Fin KielKRAUt, A. (1987). La derrota del pensamiento. Barcelona: Anagrama.

FORQUIN, J.C. (1989). Ecole et Culture. Le point de vue des sociologues britanniques. Bruselas: De Boeck.

Fou CAULT, M . (1995). Las palabras y las cosas. M adrid: Siglo XXI.

Gimen 0, J. (1991). «Currículum y diversidad cultural». Boletín del Centro de D ocumentación, 4. Valencia: Asociación de Enseñantes con Gitanos.

H ELL, V. (1994). La idea de cultura. Barcelona: Círculo de Lectores.

I GLESIAS, J. (1988). «Socialización y control social». En Tratado de Sociología, II. Salustian o del Cam Po (ed.). M adrid: Taurus.

I N GLEH ART, R. (1991). El cambio cultural en las sociedades industriales avanzadas. $M$ adrid: Siglo XXI.

Leren A, C. (1988). «Educación». En Tratado deSociología, II. Salustiano del Cam po (ed.). M adrid: Taurus.

- (1989). Escuela, ideol ogía y dases sociales en España. Barcelona: Círculo de Lectores.

M ITTER, W. (1992). «La educación multicultural: consideraciones básicas desde un punto de vista interdisciplinario». Perspectivas, vol. XXII, núm. 1 (81). Ginebra: BIE. M o Rín , E. (1988). Pensar Europa. Barcelona: G edisa.

OIE (1981). Finalidades de la educación. París: Unesco.

PASTOR, G . (1994). «G rupos minoritarios ante los medios: del pluralismo étnico a la homogeneización cultural». En Comunicación y pluralismo. AUBACH, M.T. (coord.). Salamanca: U niversidad Pontificia de Salamanca.

PARSO N S, T. (1974). «La clase como sistema social». En G RAS, A. (1980). Textosfundamentales. Sociología de la educación. M adrid: N arcea.

Sotelo, I. (1995). «Educación y D emocracia». En Volver a pensar la educación. M adrid: M orata.

SPECK, J.; W EH LE, G. (1981). Conceptos fundamentales de Pedagogía. Barcelona: H erder.

- (1993). Libro verde sobre la dimensión europea de la educación. Bruselas: Comisión de la Comunidad Europea. 